\title{
Deux modèles pour la sécularisation de l'histoire juive : Le paradigme libertin du Theophrastus redivivus et le paradigme spinoziste
}

Two models for the secularization of Jewish history: The libertine paradigm of Theophrastus redivivus and the Spinozist paradigm

Gianni Paganini*

recebido: $01 / 2013$

aprovado: $03 / 2013$

Résumé: Ce fut surtout avec l'œuvre de Flavius Josèphe, Contre Apion, que s'affirma pleinement l'image de Moïse comme chef politique et religieux à la fois, c'est-à-dire en tant que parfait "législateur" de son peuple. Flavius Josèphe fait l'éloge de la loi mosaïque, considérant qu'elle est valable à toutes les époques et non seulement dans les circonstances particulières où elle fut promulguée. Nombreux sont les manuscrits philosophiques clandestins qui entre le XVIIème et le XVIIIème siècle se présentent comme les héritiers directs de cette interprétation classico-helléniste de la religion en général et de la loi mosaïque en particulier, considérée comme " art politique ». Le Theophrastus redivivus en est un témoin exemplaire. D'autre part, il y avait une interprétation totalement alternative, dont les clandestins commenceront à disposer à partir de la publication du Traité théologicopolitique (1670) de Spinoza et surtout avec la traduction française de 1678. Cette interprétation répondait remarquablement à l'exigence de sécularisation de l'histoire juive, sans s'exposer à des appréciations plus ou moins explicitement antisémites que l'on trouve par contre dans certains manuscrits clandestins.

Mots clés: Manuscrits clandestins, Spinoza, Histoire juive, Libertinisme

Abstract: It was mainly with Flavius Josephus's work Contra Apionem that Moses's image as a political and religious leader, namely as a perfect 'legislator' of his people, was fully affirmed. Flavius Josephus praised the Mosaic law, considering it is valid at all times and not only in the particular

*Università del Piemonte Orientale (Vercelli, Italia). em@il:paganini@lett.unipmn.it.

Problemata: R. Intern. Fil. Vol. 04. No. 03. (2013), p. 109-124 ISSN 2236-8612 DOI: http://dx.doi.org/10.7443/problemata.v4i3.16258 
circumstances in which it was proclaimed. In the seventeenth and eighteenth centuries, there are abundant clandestine philosophical manuscripts that present themselves as the authentic heirs of such classical-hellenistic interpretations of the religion in general and of the Mosaic law in particular, considered as a "political art". The clandestine manuscript Theophrastus redivivus is one such exemplary testimony. On the other hand, there was a completely alternative interpretation, whose clandestine authors had at their disposal since the publication of Spinoza's work Tractatus theologicopoliticus (1670) and especially since the French translation of 1678. This interpretation responded remarkably to the secularization of the Jewish history, without maintaining more or less explicitly anti-Semitic assessments that, by contrast, we can find in some clandestine manuscripts.

Keywords: Clandestine manuscripts, Spinoza, Jewish history, Libertinism

Dans les milieux philosophiques clandestins qui ne furent pas touchés par le débat théologique interne au judaïsme du XVIIème siècle, le courant qui eut la plus grande influence sur l'image de la religion judaïque fut la tradition libertine qui considérait les fondateurs des religions comme des chefs politiques; ceux-ci, pour imposer leur «loi» aux peuples, durent recourir non seulement à la force et à la tromperie, mais aussi à l'imposture. Si les anciens avaient déjà eu l'idée d'une « religion politique » (parmi les trois types de théologie, Varron avait distingué la théologie politique de la théologie mythique et de la théologie physique), ils l'avaient cependant comprise comme un ensemble de pratiques dont on ne pourrait pas obtenir d'effets sur les dieux, mais qui devaient être respectées pour garantir la paix et la vie en commun. Ils avaient développé une critique des faux prodiges et en général des superstitions religieuses (on pense au De divinatione de Cicéron ou à l'usage politique que Numa Pompilius avait fait de la religion, en feignant d'avoir des entretiens avec la nymphe Egérie, comme on pouvait le lire dans les histoires de Titus Livius). Il y avait toutefois une différence profonde entre la tradition classique et celle judéo-chrétienne. Comme l'a noté Rémi Brague, la loi divine grecque (et par extension celle romaine) « est divine parce qu'elle exprime les structures profondes d'un ordre naturel permanent; la loi juive est divine parce qu'elle émane d'un dieu maître de l'histoire ». Pour les anciens, les dieux ne légifèrent 
pas et l'idée platonicienne (développée dans les Lois) que la ville est fondée sur un "régime divin» (theia politeia) constituait dans l'antiquité «une exception de taille»: quoi qu'il en soit, chez Platon non plus cette expression n'indique pas « une origine chez un dieu que pourrait désigner un substantif, voire un nom propre ». Pour les anciens, le divin est précisément un adjectif plutôt qu'un substantif, en référence à la loi. Il connote un peu tout: " le monde naturel et ses merveilles, y compris les immortels; ce qui, parmi les choses humaines, est là depuis toujours et se rencontre partout; et enfin ce qui dans l'homme a accès aux plus hautes réalités ». De la même façon l'idée de loi ne se présente ni comme l'«expression d'une volonté explicite », ni comme une inspiration immédiate qui trouverait sa manifestation dans une norme «divine » écrite et codifiée $^{1}$; la Grèce classique n'avait pas de « livres sacrés », ni de législateurs ou de prophètes qui pouvaient se dire directement inspirés par une «voix» divine (le statut des oracles était de fournir des prévisions, des conseils adressés à des cas et des personnes seules, et non des lois de caractère collectif pour la ville ou même pour un peuple entier) ${ }^{2}$.

Là où le divin vient qualifier une loi du monde classique, il le fait dans le cadre de ce que Jan Assmann a appelé " cosmothéisme », c'est-à-dire en tant que loi immanente au monde qui comprend aussi la Polis - tandis que la loi divine de la pensée biblique et médiévale est l'œuvre d'un dieu extérieur au monde et qu'il y intervient avec sa propre volonté et sa propre autorité. De cette façon, dans le cercle des religions " révélées », le problème théologico-politique finit par assumer un aspect complètement nouveau. Non seulement Dieu est élevé au rang de législateur et la source de son autorité est sa volonté explicite, et non un intellect (noûs) plus ou moins diffus et immanent parmi les hommes ainsi que dans le cosmos; en fait, dans les trois monothéismes, celui qui reçoit la révélation divine (comme Moïse), l'interprète (comme les prophètes bibliques), ou la met par écrit (comme dans le cas de Mahomet, à qui a été dicté le Coran), exerce les réelles fonctions du législateur, politique et religieux à la fois, puisque la révélation est essentiellement «loi » et comme telle elle doit être mise par écrit dans des livres qui deviennent pour cette raison inaltérables et «canoniques ». Evidemment le judaïsme fut la première religion où s'affirma cette nouvelle articulation théologicopolitique, profondément différente de celle classique. 
Les historiens et géographes païens, comme Hécatée d'Abdère ou Diodore de Sicile, qui furent en contact avec la culture juive, cherchèrent à comparer le rôle de Moïse à la figure qu'ils connaissaient le mieux, la figure grecque du législateur de la ville (nomothéthès), ou bien, comme Strabon, le décrivirent comme un prince égyptien rebelle. D'autre part, le judaïsme alexandrin, avec Philon, tenta une synthèse originale entre la « divinité » particulière de la loi " grecque » et celle « juive », avec comme résultat que pour Philon la loi divine devint avant tout la loi naturelle, non écrite, donc une loi universelle tournée vers l'humanité tout entière, alors que ses écrits n'étaient pas très clairs sur le rôle de Moïse : était-il lui-même un législateur ou seulement l'interprète d'un Dieu législateur ${ }^{3}$ ?

Ce fut surtout avec l'œuvre de Flavius Josèphe, Contre Apion, que s'affirma pleinement l'image de Moïse comme chef politique et religieux à la fois, c'est-à-dire en tant que parfait « législateur » de son peuple. Flavius Josèphe fait l'éloge de la loi mosaïque, considérant qu'elle est valable à toutes les époques et non seulement dans les circonstances particulières où elle fut promulguée; en même temps "il reprend maladroitement les lieux communs hellénistiques", en mettant sur le même plan Moïse et Minos, qui tous deux attribuèrent leur loi à une divinité, bien que de façons différentes, comme on a vu. Philon «envisage même l'idée selon laquelle Moïse aurait instrumentalisé le divin pour légitimer sa mission de fondateur de cité »(BRAGUE, ibid., p. 130). S'accomplit ainsi, même quant à la figure du législateur, la fusion de deux traditions très différentes, la grecque et la juive. On peut aller plus loin et dire que un législateur, 'créatif' au point de vue culturel comme Moïse, ne se limite pas à imposer une loi, mais il donne aussi un Dieu à son peuple. Ainsi que l'a écrit Arnaldo Momigliano, selon Flavius Josèphe, «ce n'est pas Dieu qui impose la Loi à Isräel par l'intermédiaire de Moïse ; c'est plutôt Moïse qui impose Dieu à Israël au moyen de la Loi » ${ }^{4}$.

Nombreux sont les manuscrits philosophiques clandestins qui entre le XVIIème et le XVIIIème siècle se présentent comme les héritiers directs de cette interprétation classicohelléniste de la religion en général et de la loi mosaïque en particulier, considérée comme « art politique ». Le Theophrastus redivivus en est un témoin exemplaire, il classe les législateurs «païens » et les législateurs monothéistes, en les unissant dans 
la catégorie des « inventeurs de la religion » en tant qu' " art de gouverner et de garder le peuple dans l'obéissance ». Ce n'est pas un hasard si le Theophrastus redivivus utilise principalement dans ce but des sources hellénistiques (Diodore de Sicile, Plutarque) ou romaines (Valère Maxime, Live). Plutarque surtout avait inauguré l'optique comparatiste par laquelle six grandes civilisations, très différentes les unes des autres, (Egypte, Crète, Sparte, Perse, les Gètes, Israël) étaient réunies par le rôle que leurs législateurs-fondateurs respectifs (Menas ou Mnevis, Minos, Lycurgue, Zarathoustra, Zalmoxis, Moïse) avaient joué en leur sein : ils surent procurer à leurs lois une stabilité durable et une autorité absolue disant qu'elles étaient suggérées par une divinité 5 . A l'époque hellénistique, nous assistons donc à un développement et à une transformation du thème 'classique' de la Theia politeia, dans le sens d'une personnalisation tant de la figure du législateur que de l'intervention de la divinité. Sous cet angle l'assimilation du personnage de Moïse est possible, même si en réalité il subsiste une différence radicale (comme nous l'avons vu) entre une loi directement dictée par Dieu et une œuvre législative vaguement inspirée, ou autorisée, ou tolérée par des contacts de différentes sortes avec la divinité.

De façon significative, c'est justement cette approche, davantage centrée sur la figure supposée divine du législateur que sur la nature de la loi, qui permet à l'auteur du Theophrastus redivivus de faire rentrer l'« exception" platonicienne dans le canon plus général de l'interprétation politique de la loi, même au prix d'un renversement audacieux de la signification des Lois. Dans ce dialogue platonicien, c'est l'athée qui s'imagine que les dieux existent uniquement en vertu de certaines lois et qu'ils sont donc la création des législateurs ${ }^{6}$; dans le Timée, ces derniers sont appelés «fils des dieux » pour indiquer justement la nature «divine» des lois qu'ils ont promulguées. Naturellement Platon condamne les « athées » et tout le dialogue des Lois inculque le respect pour le culte et la religion comme fondements indispensables de la politique. Au contraire, le Theophrastus prend à son compte le rôle de l' "athée » et s'en sert pour interpréter soit les Lois soit le passage du Timée comme une révélation «entre les lignes" du caractère entièrement et exclusivement politique de la religion. C'est pourquoi, à l'opposé de Platon, ce sont les « dieux » qui sont les 
« fils » des « lois », soit une création des législateurs qui à leur tour en sont les auteurs, et non vice et versa ${ }^{7}$.

Naturellement, cette reprise de l'image classicohellénistique du « législateur » et son application au judaïsme et à Moïse n'auraient pas été possibles sans l'intervention de la critique libertine, qui se l'était appropriée pour servir ses propres buts démythifiants, et qui en avait accentué l'aspect dévalorisant et iconoclaste. Il advint ainsi qu'en plus de s'attaquer au judaïsme, parce qu'il était la base et les racines historiques du christianisme, les libertins et les clandestins colorèrent leurs critiques d'accents antisémites, s'en prenant non seulement à l'idéologie (l'« imposture » de la religion biblique), mais aussi à la réalité du peuple juif, dans ses caractères historiques et presque raciaux. Moïse ne se soustrait pas non plus à cette œuvre de démolition systématique. Dans la culture judéohellénistique, Moïse avait été le prototype du sage législateur politico-religieux, capable de se servir des mythes, des rites et des préceptes pour ramener à l'obéissance un peuple particulièrement récalcitrant et agité. Maintenant, il subit une diminutio drastique qui touche non seulement à la signification religieuse de sa mission, clairement niée, mais aussi à la valeur politique de son œuvre. Et cette dépréciation était sensible aussi à l'égard des sources 'laïques' de sa représentation.

Par exemple, les passages où Machiavelli avait comparé Moïse aux autres législateurs païens (Cyrus, Romulus et Thésée $^{8}$, Lycurgue et Solon) ${ }^{9}$. Pour le secrétaire florentin, Moïse représentait nettement le prototype du prophète armé, du politicien rusé et prudent, qui justement pour cela ne subit pas le destin tragique d'un prophète désarmé comme Savonarole ${ }^{10}$. Machiavelli était certainement la source privilégiée de ceux qui au XVIIème siècle firent une lecture du fait religieux sous un angle politique, comme Gabriel Naudé. Celui-ci, dans le chapitre IV de ses Considérations politiques sur les coups d'estat, tout en constatant l'utilité prouvée de la «crainte des Dieux » afin de mettre un frein au "vulgaire », démontra le plein droit des législateurs à feindre toute sorte de légitimité religieuse afin d'affirmer et de consolider leur pouvoir sur le peuple. On ne devait donc pas s'étonner si les législateurs feignaient d'être en communication directe avec la divinité, imaginant de faux miracles et des prophéties présumées, en corroborant l'exercice du pouvoir avec l'éloquence du prêcheur $^{11}$. 
Naturellement, le 'cas' Moïse constituait un exemple privilégié pour l'application de cette typologie de législateurimposteur, surtout pour ce qui concernait les nombreux prodiges racontés dans L'Exode et leur possible explication magiconaturaliste. Ce type d'explication, qui faisait l'économie du transcendant et qui ramenait les présumés miracles à des effets naturels prodigieux, rares mais toujours liés à des lois naturelles occultes ou peu connues, avait déjà été avancée par un successeur d'Aristote comme Pomponazzi. Celui-ci, dans le De incantationibus, avait rappelé comment Moïse avait été considéré par des historiens antiques (Pline et Suetonius) comme «magicum et virum veneficum». Campanella, qui considérait Moïse comme un homme divinement inspiré, avait cependant rapporté, d'après les écrits de Pline, Tacite et Trogus, la thèse que «Moïse a été le plus habile des magiciens et qu'il avait réalisé tous ses miracles au moyen de la magie ». Mais c'était surtout Naudé, dans son livre significativement intitulé Apologie pour les grands hommes soupçonnez de magie, qui accomplissait la double réduction de la magie en manipulation physique et de l'inspiration divine en fiction politique. Pour Naudé, le mage n'est plus le détenteur d'un pouvoir presque surnaturel, mais seulement un savant qui connaît et qui manipule les forces cachées de la nature ; ainsi les législateurs - écrit-il « se sont servis fort à propos de ces Deitez feintes, de ces commerces supposez, de ces apparitions prétendues, et en un mot de cette Magie des anciens, pour mieux palier leur ambition et fonder plus fermement leurs Empires ${ }^{12}$. Naudé n'hésitait pas à mettre aussi en rapport ce genre d'explication naturaliste et iconoclaste avec les prodiges de Moïse et du Christ ${ }^{13}$.

Avec l'usage des sources classiques, renaissantes (parmi lesquelles Cardano, Campanella et Vanini) et libertines, le doute, qu'en réalité le judaïsme était un cas à part dans la typologie des religions comme impostures politiques, commençait à émerger chez les auteurs clandestins : pas tant pour la figure du législateur Moïse, qui pouvait facilement entrer dans la galerie plus vaste des simulateurs ambitieux, que pour les modalités et le succès de son action, qui s'expliquait uniquement en imaginant que le peuple juif était plus ignorant, crédule et arriéré que les autres. Avec l'explication politique des libertins, les auteurs des textes clandestins finirent donc par adopter une attitude manifestement anti-juive, dirigée non seulement contre la religion, mais en définitive contre toute 
l'histoire et les événements complexes vécus par le peuple juif. C'est pourquoi l'idée de l'élection divine était rejetée et réduite à un stratagème de consolation rendu nécessaire par la situation particulièrement désolante des hébreux.

Une interprétation totalement alternative, dont les clandestins commenceront à disposer à partir de la publication du Traité théologico-politique (1670) de Spinoza et surtout avec la traduction française de 1678, répondait remarquablement à l'exigence de sécularisation de l'histoire juive, sans s'exposer à des appréciations plus ou moins explicitement antisémites. Spinoza aussi avait refusé le principe selon lequel l'élection divine est réservée à un peuple unique et avait donc nié à Israël le statut de peuple bien-aimé d'un point de vue religieux ou théologique. Il lui avait toutefois reconnu une «position éminente » ("praestantia") pour le système politique de type théocratique, où « le droit civil et la religion ...étaient une seule et même chose ». En Israël la théocratie se fondait sur l'égalité des citoyens, tous soumis de façon identique au Dieu unique avec lequel ils avaient conclu un pacte, même si Moïse resta « le seul promulgateur et interprète des lois divines ». Selon Spinoza, ce fut justement Moïse qui «introduisit la religion dans la politique » ( « jussu divino religionem in Rempublican introduxit $»)^{14}$ et c'est ainsi que pour les Juifs «l'amour de la patrie » prit l'apparence de la «piété religieuse», au point de devenir « nature », avec la « haine envers les autres nations » ${ }^{15}$. C'est pourquoi, dans la république des Hébreux il n'y avait aucune distinction entre loi civile et loi religieuse. Toutefois il n'en résultait pas pour Spinoza que ce fut là la «meilleure » façon de gouverner dans l'absolu, valable pour tous les pays ${ }^{16}$; au contraire, ce système, politique et religieux à la fois, fut valable seulement pour la durée de l'état juif («stante eorum imperio $»)^{17}$.

En réalité, l'auteur du Tractatus theologico-politicus déconseillait aux autres peuples d'adopter comme modèle la forme de gouvernement de la République des Hébreux ; elle n'aurait été adaptée qu'à des peuples isolés, sans contact avec l'extérieur, prêts à se couper du reste du monde, alors qu'elle était totalement inadaptée pour des gens qui voulaient garder le contact avec les autres ${ }^{18}$. Toutefois, l'admiration du philosophe pour la signification purement "politique» de l'œuvre de Moïse était évidente ; ce n'est pas un hasard s'il attribuait 
l'échec et finalement la destruction de l'état juif à l'abandon des principes originels qui en avaient garanti la stabilité. Sur la base de ces principes, en effet, "personne n'était soumis à son semblable, mais seulement à Dieu et la charité et l'amour envers son concitoyen étaient considérés comme les devoirs religieux les plus importants ». Il serait toutefois erroné - selon Spinoza de prendre ces simples «préceptes» et ces «lois» pour une représentation appropriée de Dieu : non seulement ces lois n'avaient pas une force universelle, mais l'idée même qu'elles soient «données» par Dieu ne venait que d'une illusion anthropomorphique. Moïse « conçut Dieu comme un souverain, un législateur, un roi, comme miséricordieux, juste etc.., alors que de telles qualités sont simplement des attributs de la nature humaine et apparaissent totalement étrangères à la nature de la divinité $»{ }^{19}$.

Malgré son œuvre de sécularisation de l'histoire juive, présentée comme un " exemple» des lois de la nature humaine, mais non comme un "modèle" universel, Spinoza, toutefois, n'accepta pas la traditionnelle explication ethnologique, d'inspiration raciste avant la lettre, selon laquelle les Juifs seraient un peuple "par nature » particulièrement indiscipliné, ou inculte, ou rustre et ignorant. La nature, observe Spinoza, fait les individus et non les peuples ; ceux-ci sont formés "par les lois et les mœurs» et de ces deux choses peut découler «le caractère » d'un peuple, crée par des contingences particulières, non par sa « nature » présumée plus ou moins défavorable ${ }^{20}$.

Le philosophe n'était certes pas tendre avec les défauts du peuple hébreu, son ignorance (« rudis fere ingenii omnes erant ») et son caractère " obstiné » (« ingenium contumax quod sola vi cogi non patitur »), qui ne supporte aucune autorité ; souvent il décrivit les juifs comme des «enfants». Il donna cependant de ces caractères négatifs une interprétation qui se référait au contexte historique et social dans lequel les Israélites s'étaient trouvés avant sous le joug de l'esclavage égyptien et puis dans le désert de l'exode. N'étant plus soumis à aucune loi, les Juifs retombèrent dans un état de nature, mais en même temps ils furent incapables de se donner un "système juridique sage et d'exercer le pouvoir de façon collégiale », soit parce qu'ils étaient "usés par un pénible esclavage » qui les rendait hostiles à toute forme d'autorité, soit parce qu'ils étaient privés d'une culture propre. La réponse de Moïse à cette situation d'extrême difficulté, fut celle typique du «législateur»: 
puisque le peuple était incapable de trouver une voie d'issue rationnelle et qu'il ne supportait plus d'être soumis à une autorité humaine, à cause du souvenir du long esclavage subi sous le joug de pharaon, il eut recours à la «dévotion » religieuse, en substituant le pacte avec un souverain humain par le pacte avec Dieu, imposant donc une forme de «théocratie ».

La chose dont ils [les Hébreux] étaient le plus incapables, c'était d'établir une sage législation et d'exercer le pouvoir collectivement, puisque tous étaient de complexion grossière et déprimés par un pénible esclavage. Le pouvoir dut donc rester aux mains d'un seul, capable de commander aux autres, de les contraindre par la force et enfin de prescrire des lois et les interpréter [...] mais il mit tous ses soins à ce que le peuple fit son devoir non pas par crainte mais de son plein gré. Deux raisons principales le contraignaient à agir de la sorte : l'insoumission naturelle du peuple (qui ne souffrait pas d'être dominé par la force seule) et l'imminente menace de la guerre [...]. Pour cette raison Moïse, par sa veru divine et sur ordre divin, introduisit la religion dans l'Etat afin que le peuple fit son devoir plus par dévotion que par crainte (ibid., V, p. 131).

Donc, pour Spinoza Moïse ne fut pas un «imposteur », mais un législateur qui sut adapter les exigences du gouvernement politique à la situation historique de son peuple ; les Hébreux ne furent pas "par nature" ni inférieurs, ni supérieurs (parce qu'élus) aux autres peuples. Simplement, ils se trouvèrent dans une situation par de nombreux aspects exceptionnelle ; ils furent précipités dans un état de nature, mais à partir d'une condition présentant des désavantages extrêmes, c'est-à-dire l'esclavage et la privation de toute culture et de toute liberté. Ces mêmes raisons expliquaient, pour Spinoza, le fait que la loi cérémoniale de Moïse, à la différence des autres législations moins contraignantes, contrôlait rigidement presque tous les aspects et les détails de la vie, tant collective qu'individuelle. Le peuple juif était incapable de se gouverner lui-même, rationnellement, et il avait besoin de prescriptions précises inculquées selon l'idée de l'ordre divin.

Les commandements de Moïse ne furent pas donnés comme des «prophéties» mais bien comme des normes; au contraire l'enseignement de Jésus ne fut pas, selon Spinoza, une « loi », mais une "doctrine morale », et le Christ ne s'occupa pas d'autre chose « que de donner des enseignements moraux et 
de les garder distincts des lois de l'Etat ». C'est pourquoi Jésus «n'abrogea en rien la loi de Moïse» (ibid., p. 70-1). Pour Spinoza le prototype du législateur-imposteur n'était donc ni Moïse (dont il fournissait une interprétation somme toute «bienveillante», bien que limitée au contexte historique « local» des Hébreux), ni le Christ, qui ne donna aucune loi, mais plutôt Mahomet. Comme le déclara le philosophe dans une lettre à Ostens : "il est clair que, d'après mes principes, il [Mahomet] fut un imposteur, puisqu'il abolit entièrement la liberté que la Religion Universelle, révélée par la lumière naturelle prophétique, permet et qui doit être pleinement autorisée comme je l'ai démontré $»^{21}$.

Parallèlement, l'explication que Spinoza donna dans le chapitre XVII du Traité théologico-politique de la décadence et de la dissolution de la république des Hébreux faisait appel à des raisons toutes politiques et négligeait toute considération relative à la supposée "nature » des Hébreux. Le philosophe mettait en cause avant tout l'infraction au pacte constitutif avec Dieu, considéré comme le souverain de la théocratie d'Israël, et le passage au régime résolument «humain» de la royauté; il mentionnait ensuite la rivalité entre les tribus et le fait que les Lévites constituèrent «un état dans l'état», provocant la jalousie et la rivalité dans le peuple; enfin il montra les effets désastreux produits par l'opinion que Moïse aurait agi non par « ordre divin » mais « selon son bon plaisir», dans l'intention de favoriser sa tribu ${ }^{22}$.

$\mathrm{Au}$ delà des références au pacte avec Dieu, qui pour Spinoza avait essentiellement valeur de «contrat social», le schéma exposé dans le Traité avait le mérite de laïciser le statut d'Israël, en excluant toute position privilégiée qui n'était pas d'ordre strictement politique. De plus, cet avantage aussi était limité, comme on a vu, à un peuple isolé et séparé des autres, non pas parce qu'il était supérieur, mais parce que les circonstances, la position, les rapports de force l'obligeaient à éviter les contacts avec des peuples plus forts et des empires menaçants. De cette manière, Spinoza sauvegardait de nombreux aspects positifs de la figure de Moïse en tant que législateur, même s'il délimitait sa signification à un contexte étréci et historiquement déterminé ; il pouvait surtout ainsi exclure du discours sur la "république des Hébreux» tous les facteurs de nature « raciale $»$. 
Sur ce thème, le chapitre III du Traité théologicopolitique est fondamental. Dans ce chapitre, l'auteur, après avoir rappelé que les «nations » se distinguent entre elles seulement " par rapport au type de société et aux lois sous lesquelles elles vivent ", souligne le fait que l' "élection et la vocation» des Hébreux consistent uniquement « dans le bonheur temporaire et le bien-être de la vie civile» (" in sola imperii temporanea foelicitate et commodis $\rangle)$, alors qu'en ce qui concerne l'« intellect » ils sont « égaux aux autres gens », à l'exception de très peu de sages. Donc "l'élection des Juifs» ne se rapporta pas à autre chose qu'à « la prospérité matérielle temporaire et à la liberté, soit à l'Etat politique » et par conséquent aux « lois », alors que «en toutes les autres choses en lesquelles consiste le vrai bonheur de l'homme, ils furent égaux à tous les autres » (ibid., III, p. 47-9).

Spinoza allait même jusqu'à affirmer que «chaque Juif, considéré en soi, en dehors de la société et de l'Etat, ne jouit d'aucun don divin parce que préféré à d'autres, et qu'il n'existe aucune différence entre lui et un païen » (ibid. p. 50). Le don même de la prophétie fut commun aussi aux autres nations, contrairement à l'opinion des Pharisiens qui dans le cas des païens l'attribuaient à «je ne sais quelle diabolique vertu». Quant au fait que les Hébreux sont restés différents en tant que peuple même après la chute de l'état, cela ne dépendait pas selon Spinoza d'une spéciale et "éternelle " élection (cette dernière, considérée comme «éternel pacte de la connaissance et de l'amour de Dieu, est universelle ») mais plutôt du fait d'avoir accentué leur « isolement» au point d' "attirer contre eux la haine de tous les gens ». Les différentes expériences d'intégration forcée des Juifs (par conversion en Espagne et par dissimulation ou marranisme au Portugal) démontraient, aux yeux de Spinoza, que c'était paradoxalement «la haine des nations » qui les confortait dans leur isolement. Cela démontrait que là où l'exclusion diminuait suite à la conversion (l'Espagne) les juifs restés sur place s'étaient intégrés dans la société chrétienne, tandis que là où la ségrégation s'était maintenue (Portugal) elle avait produit un isolement constant, bien que celé par la dissimulation marrane. Donc, la prétention alléguée par les Hébreux de "se considérer supérieurs aux autres peuples » était rendue vaine par Spinoza, sauf pour ce qui concernait le système politique tant qu'il existât ; et même, l'isolement de 
«privilège » se transformait historiquement en «stigmate», avec pour seul effet «positif» le fait qu' on lui doit la survivance de l'identité juive même dans les conditions de persécution ${ }^{23}$.

Enfin, bien que n'étant pas tendre avec l'ignorance et la crédulité, dont il soulignait la présence constante dans l'histoire d'Israël, Spinoza les ramenait toutes les deux vers des aspects généraux de la psychologie humaine, dominée par la force de l'imagination ; sous cet aspect, les Israëlites n'étaient pour lui ni meilleurs ni « pires » que les autres peuples, quand en raison de leur ignorance des causes naturelles ils attribuaient à la puissance divine les phénomènes dont ils ignoraient l'explication les appelant miracles. Il n'y avait donc pas pour Spinoza une spécificité « éthnique », ni en positif ni en négatif, des Israëlites par rapport aux autres gens ; selon le philosophe, leurs caractères dépendaient soit de constantes typiques de la nature humaine et furent donc communs à tous les peuples, soit, quand ils étaient distinctifs du seul peuple juif, on pouvait les faire remonter aux événements politiques qui en avaient modelé les comportements et la façon de penser.

Popkin a avancé l'hypothèse (abandonnée par la suite) que Spinoza pouvait avoir été impliqué directement ou indirectement, " volontairement ou involontairement », dans la première phase d'élaboration du Traité des trois imposteurs ${ }^{24}$. Indépendamment de cette conjecture, très hazardeuse et qui, en réalité, n' a trouvé aucune confirmation dans les études sur la rédaction du traité clandestin, il reste le fait - bien documenté par Popkin - que l'auteur du Tractatus theologico-politicus non seulement était pleinement au courant de la thèse de l'imposture des religions, mais que par de nombreux aspects sa doctrine constituait une réponse à cette thèse. En effet, d'une part le philosophe alla bien au-delà de l'explication purement politique $\mathrm{du}$ phénomène religieux, mais d'autre part il contribua à la sécularisation de l'histoire juive, en la ramenant à des événements historiques particuliers ou à des données psychologiques et passionnelles universelles. En ce qui concerne le premier aspect (l'usage politique de la crainte religieuse), Spinoza l'énonça clairement dans la préface du Tractatus, même s'il en limita l'emploi aux «monarchies » absolues et qu'il exclut qu'il put avoir une influence sur les « libres républiques ». Selon lui, c'est dans les premières que s'accomplit cette exploitation politique des croyances religieuses consistant «à tromper les hommes et voiler par le nom spécieux de religion la 
crainte qui sert à les freiner ». Les hommes, observe Spinoza, sont les victimes de la superstition quand ils vivent dans le giron de la peur et, comme l'avait déjà dit l'historien romain Quintus Curtius, « rien ne réussit mieux que la superstition à dominer les masses ». En poursuivant, le philosophe avait noté que ce sont bien les monarchies qui poussent les peuples «à adorer comme des dieux leurs rois", alors que dans les républiques "il est contraire à la liberté collective d'étouffer avec des préjugés ou de contraindre d'une façon ou d'une autre la libre opinion individuelle $»^{25}$.

La théorie libertine de l'imposture religieuse avait donc une certaine validité même pour Spinoza, mais elle s'appliquait principalement aux gouvernements absolus. En ce qui concerne, par contre, le second aspect (l'explication au moyen de données historiques ou passionnelles), la synthèse la plus efficace de la position de Spinoza est celle esquissée par P.-F. Moreau dans son essai sur « Spinoza et l'Etat des Hébreux » :

Comme la nature humaine est toujours et partout la même, il n'existe ni moment historique privilégié, ni peuple plus près de Dieu que les autres; l'histoire biblique ne nous montre d'ailleurs pas une véritable préséance de l'Eglise sur le Magistrat; et elle se divise en époques qui, si elles tiennent leur unité de paraître comme l'accomplissement différé d'une erreur initiale [1'attribution du sacerdotat au Lévites, alors que Moïse l'avait réservé aux aînés de chaque famille de toutes les tribus], n'en ont pas moins chacune son visage propre ${ }^{26}$.

\section{NOTES}

\footnotetext{
${ }^{1}$ Rémi Brague, La loi de Dieu. Histoire philosophique d'une alliance, Paris, 2005, p. 31, 40-44.

2 Pour le rapport essentiel entre la révolution monothéiste juive, la désincarnation de la loi à partir de son incarnation réelle (le souverainlégislateur) et sa traduction en " écriture », à son tour codifiée et canonisée, voir les observations de Jan Assmann, Of God and Gods : Egypt, Israel, and the rise of monotheism, Madison, 2008, chap. V (cf. p. 137: « La Torah remplace le souverain-législateur dans ses deux fonctions, en tant que source de la loi et en tant qu'interface entre loi et histoire. C'est là une particularité de la codification légale biblique. En Grèce, en effet, la loi codifiée n'est ni insérée dans une histoire normative ni attribuée à la divinité comme source de la législation »). Voir aussi Id. et Aleida Assmann (éds), Kanon und Zensur, Müunchen, 1987.

${ }^{3}$ Nous suivons Brague, La loi de Dieu, p. 128-129.
} 
${ }^{4}$ Arnaldo Momigliano, «Un'apologia del giudaismo: il Contro Apione di Flavio Giuseppe ». Sur l'image de Moïse dans la culture du XVIIIème siècle cf. Wolf D. Hartwich, Die Sendung Moses. Von der Aufklärung bis Thomas Mann, München, 1997.

${ }^{5}$ Diodorus Siculus, Bibliotheca Historica, I, 94, 1-2, cité par le Theophrastus redivivus (éd. G. Canziani et G. Paganini, Firenze, 1981-1982), p. 350. En suivant Valerius Maximus le Theophrastus étend ultérieurement la liste des législateurs divinement inspirés.

${ }^{6}$ Platon, Leges, X, 889 e 4

${ }^{7}$ Cf. Theophrastus redivivus, p. 349-357. Pour le passage du Timée : XIII, 40 d-e.

${ }^{8}$ Niccolò Machiavelli, Il Principe, VI, (in Il principe e Discorsi, a cura di Sergio Bertelli, Milano, 1960, p. 30).

${ }^{9}$ Machiavelli, Discorsi sopra la prima deca di Tito Livio, I, ix, ibid. p. 154. Le thème est repris par Machiavel dans le fondamental chap. xi («Della religione de' Romani »), p. 160-163, amplement cité d'ailleurs dans le Theophrastus redivivus, p. 357-359.

${ }^{10}$ Machiavelli, Il Principe, VI, p. 32: «C'est pour cela qu'on a vu réussir tous les prophètes armés, et finir malheureusement ceux qui étaient désarmés. Sur quoi l'on doit ajouter que les peuples sont naturellement inconstants, et que, s'il est aisé de leur persuader quelque chose, il est difficile de les affermir dans cette persuasion : il faut donc que les choses soient disposées de manière que, lorsqu'ils ne croient plus, on puisse les faire croire par force. Certainement Moïse, Cyrus, Thésée et Romulus n'auraient pu faire longtemps garder leurs institutions, s'ils avaient été désarmés; et ils auraient eu le sort qu'a éprouvé de nos jours le frère Jérôme Savonarole, dont toutes les institutions périrent aussitôt que le grand nombre eut commencé de ne plus croire en lui, attendu qu'il n'avait pas le moyen d'affermir dans leur croyance ceux qui croyaient encore, ni de forcer les mécréants à croire ». Ce passage est repris aussi par l'auteur du Theophrastus redivivus, p. 360.

${ }^{11}$ Gabriel Naudé, Considérations politiques sur les coups d'estat, s.1., sur la copie de Rome, 1667, p. 243-244, 251-251, 256.

12 Naudé, Apologie pour les grands hommes soupçonnés de magie, IV, Amsterdam, Humbert, 1712, p. 32-33. Cf. Le chapitre IV dont le titre est : "Que la grande doctrine de plusieurs hommes a été souvent prise pour Magie » (p. 38 sqq.)

${ }^{13}$ Ibid., II, p. 25-26. Sur la théorie de l'imposture sacerdotale du XVIIIème siècle, cf. Frank Manuel, The Eighteenth Century confronts the Gods, Cambridge (Mass.), 1959, p. 47-53.

14 Spinoza, Tractatus Theologico-Politicus, V (Opera, éd. Carl Gebhardt, Heidelberg 1927, t. III, p. 75).

${ }^{15}$ Ibid., XVII, p. 201 sqq.

${ }^{16} \mathrm{Ibid}, \mathrm{IV}, \mathrm{p} .68$.

${ }^{17}$ Ibid., V, p. 74.

${ }^{18}$ Ibid.,. XVIII, p. 221-228.

${ }^{19} \mathrm{Ibid}$., IV, p. 63-64. Sur la nouveauté de Spinoza par rapport au mainstream de l'interprétation de la république des Hébreux en tant que modèle politique entre les XVIème et XVIIème siècles, voir Eric Nelson, The Hebrew Republic. Jewish Sources and the Transformation of European Political 
Thought, Cambridge (Mass.)-London, 2010, p. 130-134. Plus général : C. R. Ligota, "Histoire à fondement théologique: La République des Hébreux » in L'Ecriture Sainte au temps de Spinoza et dans le systême spinoziste, Paris, 1992, p. 149-167.

${ }^{20}$ Spinoza, Tractatus, cap. XVII, p. 206-207, 215, 217: «At forsan hîc aliquis dicet, id evenisse ex gentis contumacia. Verum hoc puerile est; nam cur haec nation reliquis contumacior fuit? An natura? Haec sane nations non creat, sed individual, quae quidem in nations non distinguuntur nisi ex diversitate linguae, legume et morum receptorum, et ex his duobus, legibus scilicet et moribus, tantum oriri potest, quod unaquaeque nation singular habeat ingenium, singularem conditionem et denique singularia praeiudicia. $\mathrm{Si}$ igitur concedendum esset, quod Hebraei supra reliquos mortals contumacies fuerint, id vitio legume vel receptorum morum imputari deberet $\gg$ (p. 217).

${ }^{21}$ Spinoza, lettre à Jacob Ostens, février 1671, Ep. 43 (Opera, t. IV p. 226)

${ }^{22}$ Spinoza, Tractatus, p. 217-221.

23 Ibid., p. 56-57. N. Wachtel, dans son beau livre sur le phénomène «marrane» (La Foi du souvenir. Labyrinthes marranes, Paris, 2001, Introduction: "Marranisme et modernité ») a souligné le caractère « novateur, et même subversif » de cette explication qui pour la première fois refuse une quelconque intervention de Dieu dans l'histoire humaine et procède à la sécularisation de l'histoire juive. Mais pour plus de détails voir Hayim Yerushalmi, Sefardica. Essais sur l'histoire des Juifs, des marranes et des nouveaux chrétiens d'origine hispano-portugaise, Paris, 1998, p. 175206 : «Propos de Spinoza sur la survivance du peuple juif»

${ }^{24}$ R. H. Popkin, «Spinoza and the Three Impostors », In. Edwin Curley et Pierre-François Moreau (éds), Spinoza. Issues and Directions. The Proceedings of the Chicago Spinoza Conference, Leiden, 1980, p. 347-358; voir ensuite Id., «Spinoza and Bible Scholarship », in Don Garrett (éd.), Cambridge Companion to Spinoza, Cambridge, 1996, p. 383-407; Hugh B. Nisbet, "Spinoza und die Kontroverse De tribus impostoribus », in K. Gründer et W. Schmitt-Biggemann (éds), Spinoza in der Frühzeit seiner religiösen Wirkung, Heidelberg, 1984, p. 227-244.

${ }^{25}$ Spinoza, Tractatus theologico-politicus, Praefatio (in Opera, IV, 1925, p. 6-7).

${ }^{26}$ Pierre-François Moreau, Spinoza. Etat et religion, Paris, 2005, p. 28. 